\title{
Submersive Rational Difference Systems and Their Accessibility
}

\author{
Miroslav Halás ${ }^{1}$, Ülle Kotta ${ }^{2 *}$ Ziming Li ${ }^{3} ;$ Huaifu Wang ${ }^{3}$, and Chunming Yuan ${ }^{3}$ \\ ${ }^{1}$ Inst. of Control and Industrial Informatics, Fac. of Electrical Engineering \& Information Technology \\ Slovak Univ. of Technology, Ilkovičova 3, 81219 Bratislava, Slovakia \\ miroslav.halas@stuba.sk \\ 2 Inst. of Cybernetics, Tallinn Univ. of Technology, Akademia tee 21, 12618 Tallinn, Estonia \\ kotta@cc.ioc.ee \\ ${ }^{3}$ Key Lab of Math. Mech., AMSS, Chinese Academy of Sciences, Beijing (100190), China \\ \{zmli,wanghf,cmyuan\}@mmrc.iss.ac.cn
}

\begin{abstract}
The paper describes an algebraic construction of the inversive difference field associated with a discrete-time rational nonlinear control system under the assumption that the system is submersive. We prove that a system is submersive iff its associated difference ideal is proper, prime and reflexive. Next, we show that Kähler differentials of the above inversive field define a module over the corresponding ring of Ore operators, and relate its torsion submodule to the vector space of autonomous one-forms, introduced elsewhere. The above results allow us to check accessibility property and simplify transfer functions with computer algebra techniques.
\end{abstract}

Categories and Subject Descriptors:

I.1.2 [Computing Methodologies]: Symbolic and Algebraic Manipulation - Algebraic Algorithms

General Terms: Algorithms, Theory

Keywords: rational difference system, submersivity, accessibility, reflexive ideal, inversive closure, transfer function

\section{INTRODUCTION}

Many algebraic concepts such as Ore (skew) polynomial rings, modules over skew polynomial rings, difference fields and ideals play a prominent role in (nonlinear) control theory. Their applications, in general, require the help of computer algebra software. In Maple there exists a built-in package, OreTools [1], that addresses the computations with Ore polynomials. Unfortunately, this package, though being a good starting point for control applications, cannot handle the Ore polynomials associated to nonlinear control systems without further extension. The reason is that one cannot describe the relations between control system variables explicitly

*sponsored in part by Estonian Science Foundation grant N 6922.

${ }^{\dagger}$ Z. Li, H. Wang and C.M. Yuan are sponsored in part by a 973 key project (no. 2004CB318000) and an NSFC project (no. 76596100).

Permission to make digital or hard copies of all or part of this work for personal or classroom use is granted without fee provided that copies are not made or distributed for profit or commercial advantage and that copies bear this notice and the full citation on the first page. To copy otherwise, to republish, to post on servers or to redistribute to lists, requires prior specific permission and/or a fee.

ISSAC'09, July 28-31, 2009, Seoul, Republic of Korea.

Copyright 2009 ACM 978-1-60558-609-0/09/07 ...\$10.00. in this package, as well as take these relationships into account in calculations with Ore polynomials.

The extension of the package so that it can address the control problems, is not immediate and trivial, especially for discrete-time systems. The main goal of this paper is to build a solid mathematical ground that will help to implement the required changes.

An Ore (called alternatively pseudo-linear) algebra approach has been applied in [15] for a unification of the study of nonlinear control systems, both discrete- and continuous-time. Mathematically speaking, the approach consists of three steps. Namely, one first associates a differential or inversive difference field to a continuousor discrete-time nonlinear control system, respectively; second, one applies the differential operator to "linearize" the given system, yielding the system description in terms of differential one-forms; and finally, one introduces pseudo-linear operators acting on differential one-forms. This approach allows us to handle different control problems, including accessibility, system equivalence and reduction [19], realization [20], feedback linearization [2]. Furthermore, this approach enables us to extend the concept of the transfer functions for nonlinear systems [14, 15, 16, 26].

Although the last two steps can be carried out in a similar manner both for continuous- and discrete-time systems, it is much easier to associate a field to a continuous-time system than to a discretetime system. Note that for a continuous-time system, such a field is proved to exist and its construction is given in [9] using differential algebra. For a discrete-time system of the form

$$
\Sigma:\left\{\begin{array}{ccc}
x_{1}(t+1) & = & f_{1}\left(u_{1}(t), \ldots, u_{m}(t), x_{1}(t), \ldots, x_{n}(t)\right) \\
& \ldots & \\
x_{n}(t+1) & = & f_{n}\left(u_{1}(t), \ldots, u_{m}(t), x_{1}(t), \ldots, x_{n}(t)\right),
\end{array}\right.
$$

such a field exists, if the system is generically submersive, that is, if the Jacobian matrix $\frac{\partial\left(f_{1}, \ldots, f_{n}\right)}{\partial\left(u_{1}, \ldots, u_{m}, x_{1}, \ldots, x_{n}\right)}$ has rank $n$ almost everywhere, except on a set of measure zero [13, 2, 15]. In this case, the field has been constructed in [2], using the tools both from algebra and classical analysis.

The main focus of this paper is to provide an algebraic construction of the inversive difference field associated with $\Sigma$, when all $f_{1}, f_{2}, \ldots, f_{n}$ are rational functions. This paves a way to apply computer algebra techniques (see for example $[1,5]$ ) for the last two steps. We show that $\Sigma$ corresponds to a proper, prime and reflexive ideal iff it is submersive. From a technical point of view, completing the second step, we apply the notion of Kähler differentials which has been widely used in control theory to study the structural properties of the nonlinear systems; see for instance [10] 
and references therein. We show that Kähler differentials of the elements of the inversive field, associated with $\Sigma$, define a module over the corresponding ring of Ore (pseudo-linear) operators, and relate its torsion submodule to the vector space of autonomous oneforms, introduced in [2,19]. Finally, we apply these constructions to check accessibility (controllability) and simplify transfer functions. Similar results are obtained for an input-output system description: $y(t+n)=f(u(t), \ldots, u(t+n-1), y(t), \ldots, y(t+n-1))$, where $f$ is a rational function.

The rest of this paper is organized as follows. In Section 2, we define the submersivity property of rational difference systems, and connect submersivity with algebraic independence. In Section 3, we prove that a rational difference system is submersive iff its associated ideal is proper, prime and reflexive, and define its associated field whose inversive closure is constructed explicitly in Section 4. Kähler differentials are described in module-theoretic language in Section 5. Similar results for rational difference equations of any order are given in Section 6. We discuss how to define and check accessibility property, and simplify transfer functions in Section 7.

\section{A DIFFERENCE FIELD EXTENSION}

In this section, we connect submersivity property with algebraic independence, and construct a difference field containing a solution of a given submersive rational system.

Let us recall some terminologies from difference algebra. The reader is referred to [8] for more details. Let $R$ be a commutative ring, and $\sigma: R \rightarrow R$ be a monomorphism, i.e. an injective homomorphism. The pair $(R, \sigma)$ is called an (ordinary) difference ring. It is called a difference domain (resp. a difference field) if it is an integral domain (resp. a field). If $\sigma$ is bijective, then $R$ is said to be inversive. Let $\left(R_{1}, \sigma_{1}\right)$ and $\left(R_{2}, \sigma_{2}\right)$ be two difference rings. We say that $R_{2}$ is a difference ring extension of $R_{1}$ if $R_{1} \subset R_{2}$ and $\left.\sigma_{2}\right|_{R_{1}}=\sigma_{1}$.

Throughout the paper, $(k, \sigma)$ stands for an inversive difference field of characteristic zero. Let $\mathbf{u}=\left\{u_{1}, \ldots, u_{m}\right\}$ be a set of $m$ indeterminates, and $\mathbf{U}=\left\{\sigma^{\ell}(u) \mid u \in \mathbf{u}, \ell \in \mathbb{N}\right\}$ an infinite set of indeterminates containing $\mathbf{u}$. We extend $\sigma$ from $k$ to $k(\mathbf{U})$ by mapping $u$ to $\sigma(u)$ for all $u \in \mathbf{U}$. Then $(k(\mathbf{U}), \sigma)$ becomes a difference field. Note that $\sigma$ is not surjective, because none of the $u_{i}$ has preimages.

Let $\mathbf{x}=\left\{x_{1}, \ldots, x_{n}\right\}$ be a set of new indeterminates. By a rational difference system over $k$, we mean a system of the form

$$
\Sigma:\left\{\sigma\left(x_{1}\right)=f_{1}, \ldots, \sigma\left(x_{n}\right)=f_{n}\right\}
$$

where $f_{1}, \ldots, f_{n}$ are in $k(\mathbf{U}, \mathbf{x})$. Following $[13,2]$, we say that (1) is submersive if the $n \times(m+n)$ matrix

$$
\frac{\partial\left(f_{1}, \ldots, f_{n}\right)}{\partial\left(u_{1}, \ldots, u_{m}, x_{1}, \ldots, x_{n}\right)}, \quad \text { abbreviated as: } \frac{\partial\left(f_{1}, \ldots, f_{n}\right)}{\partial(\mathbf{u}, \mathbf{x})},
$$

has rank $n$.

System (1) has a solution in some difference field extension of $k$ if the following question has an affirmative answer:

Given $f_{1}, \ldots, f_{n} \in k(\mathbf{U}, \mathbf{x})$, can $\sigma$ be extended to a monomorphism $\theta$ from $k(\mathbf{U}, \mathbf{x})$ to $k(\mathbf{U}, \mathbf{x})$ with $\theta\left(x_{j}\right)=f_{j}$ for all $j$ with $1 \leq j \leq n$ ?

First, we extend $\sigma: k(\mathbf{U}) \rightarrow k(\mathbf{U})$ to $\theta: k(\mathbf{U})[\mathbf{x}] \rightarrow k(\mathbf{U}, \mathbf{x})$ by mapping $x_{j}$ to $f_{j}$ for all $j$ with $1 \leq j \leq n$. The homomorphism $\theta$ can be extended further to the fraction field $k(\mathbf{U}, \mathbf{x})$, provided that it is injective. Observe that $\theta$ is injective iff any nonzero polynomial in $k(\mathbf{U})[\mathbf{x}]$ is not in its kernel, which is equivalent to that

$$
\sigma(\mathbf{U}) \cup\left\{\theta\left(x_{1}\right), \ldots, \theta\left(x_{n}\right)\right\}, \quad \text { i.e. } \quad \sigma(\mathbf{U}) \cup\left\{f_{1}, \ldots, f_{n}\right\}
$$

is algebraically independent over $\sigma(k)$. Note that $\sigma(k)=k$, since $\sigma$ is an automorphism. So we seek a criterion for the algebraic independence of $\sigma(\mathbf{U}) \cup\left\{f_{1}, \ldots, f_{n}\right\}$ over $k$.
To do this, we need the notion of Kähler differentials [18]. A tensor-free definition of Kähler differentials is given in [28], and basic results about Kähler differentials are contained in [3, §9.1]. Applications of Kähler differentials in nonlinear control theory are given in [10] and references therein.

For two fields $F \subset E$, there exist a linear space $\Omega_{E / F}$ over $E$, and a derivation $\mathrm{d}: E \rightarrow \Omega_{E / F}$ such that (i) $\mathrm{d} c=0$ for all $c \in F$; and (ii) $\Omega_{E / F}=\operatorname{span}_{E}\{\mathrm{~d} a \mid a \in E\}$, where $\mathrm{d} a$ denotes the image of $a$ under d. An element of $\Omega_{E / F}$ is called an $F$-differential, and the pair $\left(\Omega_{E / F}, \mathrm{~d}\right)$ is called the $E$-linear space of $F$-differentials. The next lemma is important for this paper. Its proof can be found in $[18,28]$ and [3, Corollary 9.1.1].

Lemma 2.1 Let $F \subset E$ be fields of characteristic zero. Then $P \subset E$ is algebraically independent over $F$ iff $\{\mathrm{d} p\}_{p \in P} \subset \Omega_{E / F}$ is linearly independent over $E$.

Corollary 2.2 In $\Omega_{k(\mathbf{U}, \mathbf{x}) / k}$, the subset $\{\mathrm{d} z \mid z \in \mathbf{U} \cup \mathbf{x}\}$ is linearly independent over $k(\mathbf{U}, \mathbf{x})$.

Proof. Observe that $\mathbf{U} \cup \mathbf{x}$ is algebraically independent over $k$, and then apply Lemma 2.1.

The algebraic independence of $\sigma(\mathbf{U}) \cup\left\{f_{1}, \ldots, f_{n}\right\}$ is equivalent to submersivity of (1), as stated in the next lemma.

Lemma 2.3 Let $f_{1}, \ldots, f_{n}$ be in $k(\mathbf{U}, \mathbf{x})$. Then $\sigma(\mathbf{U}) \cup\left\{f_{1}, \ldots, f_{n}\right\}$ is algebraically independent over $k$ iff $\frac{\partial\left(f_{1}, \ldots, f_{n}\right)}{\partial(\mathbf{u}, \mathbf{x})}$ has rank $n$.

Proof. For brevity, we denote $\frac{\partial\left(f_{1}, \ldots, f_{n}\right)}{\partial(\mathbf{u}, \mathbf{x})}$ by $\mathcal{I}$. Let $(\Omega, \mathrm{d})$ be the linear space of $k$-differentials over $k(\mathbf{U}, \mathbf{x})$,

$$
L=\{\mathrm{d} v \mid v \in \sigma(\mathbf{U})\} \cup\left\{\mathrm{d} f_{1}, \ldots, \mathrm{d} f_{n}\right\},
$$

and $V$ be the $k(\mathbf{U}, \mathbf{x})$-linear subspace spanned by $\{\mathrm{d} v \mid v \in \sigma(\mathbf{U})\}$. By Lemma 2.1, it suffices to show that $L$ is linearly dependent over $k(\mathbf{U}, \mathbf{x})$ iff $\operatorname{rank}(\mathcal{I})<n$. A straightforward calculation yields

$$
\left(\mathrm{d} f_{1}, \ldots, \mathrm{d} f_{n}\right)^{T} \equiv \mathcal{I}\left(\mathrm{d} u_{1}, \ldots, \mathrm{d} u_{m}, \mathrm{~d} x_{1}, \ldots, \mathrm{d} x_{n}\right)^{T} \quad \bmod V,
$$

where $(\cdot)^{T}$ stands for the transpose of a matrix (vector).

If the rank of $\mathcal{I}$ is less than $n$, then there exists a nonzero vector $\left(r_{1}, \ldots, r_{n}\right)$ in the left kernel of $\mathcal{J}$. Thus, $\sum_{j=1}^{n} r_{j} \mathrm{~d} f_{j}$ belongs to $V$. So $L$ is linearly dependent over $k(\mathbf{U}, \mathbf{x})$. Conversely, suppose that $L$ is linearly dependent over $k(\mathbf{U}, \mathbf{x})$. It follows from Corollary 2.2 that $\{\mathrm{d} v \mid v \in \sigma(\mathbf{U})\}$ is linearly independent over $k(\mathbf{U}, \mathbf{x})$. So a nontrivial linear relation among elements in $L$ implies that there exist $r_{1}, \ldots, r_{n}$ in $k(\mathbf{U}, \mathbf{x})$, not all zero, such that $\sum_{j=1}^{n} r_{j} \mathrm{~d} f_{j}$ belongs to $V$. Multiplying $\left(r_{1}, \ldots, r_{n}\right)$ to (2) yields

$$
\left(r_{1}, \ldots, r_{n}\right) \mathcal{I}\left(\mathrm{d} u_{1}, \ldots, \mathrm{d} u_{m}, \mathrm{~d} x_{1}, \ldots, \mathrm{d} x_{n}\right)^{T} \in V .
$$

Thus, $\left(r_{1}, \ldots, r_{n}\right)$ is in the left kernel of $\mathcal{J}$, for, otherwise, the subset $\{\mathrm{d} z \mid z \in \mathbf{U} \cup \mathbf{x}\}$ would be linearly dependent, a contradiction to Corollary 2.2. The rank of $\mathcal{I}$ is less than $n$.

The next proposition is immediate from Lemma 2.3.

Proposition 2.4 Let $f_{1}, \ldots, f_{n}$ be in $k(\mathbf{U}, \mathbf{x})$, and $\theta$ be the homomorphism from $k(\mathbf{U})[\mathbf{x}]$ to $k(\mathbf{U}, \mathbf{x})$ such that $\left.\theta\right|_{k}=\sigma, \theta(u)=\sigma(u)$ for all $u \in \mathbf{U}$ and $\theta\left(x_{j}\right)=f_{j}$ for all $j$ with $1 \leq j \leq n$. Then $\theta$ is injective iff the matrix $\frac{\partial\left(f_{1}, \ldots, f_{n}\right)}{\partial(\mathbf{u}, \mathbf{x})}$ has rank $n$. When this is the case, $\theta$ can be extended to a monomorphism from $k(\mathbf{U}, \mathbf{x})$ to itself, that is, $(k(\mathbf{U}, \mathbf{x}), \theta)$ is a difference field extension of $(k, \sigma)$.

System (1) has a solution $(\mathbf{u}, \mathbf{x})$ in the difference field defined in Proposition 2.4, provided that (1) is submersive. 


\section{ASSOCIATED DIFFERENCE IDEALS}

In this section, we characterize a submersive system by a proper, prime and reflexive ideal, and show that the field associated with the system is isomorphic to $(k(\mathbf{U}, \mathbf{x}), \theta)$ defined in Proposition 2.4.

Let $(R, \sigma)$ be a difference ring and $I$ an ideal of $R$. We say that $I$ is proper if $I \neq R$, and that it is a difference ideal if $\sigma(I) \subset I$.

Assume now that $I$ is a difference ideal. Then $I$ is said to be reflexive if, for every $r \in R, \sigma(r) \in I$ implies that $r \in I$. The induced homomorphism $\bar{\sigma}: R / I \rightarrow R / I$ with $r+I \mapsto \sigma(r)+I$ for all $r \in R$, is injective iff $I$ is reflexive. In other words, $(R / I, \bar{\sigma})$ is a difference ring iff $I$ is reflexive. A homomorphism $\phi$ from $\left(R_{1}, \sigma_{1}\right)$ to $\left(R_{2}, \sigma_{2}\right)$ is called a difference homomorphism if $\phi \circ \sigma_{1}=\sigma_{2} \circ \phi$. The kernel of a difference homomorphism is proper and reflexive by Theorem $\mathrm{V}$ in [8, page 71$]$.

Lemma 3.1 Let $\phi$ be a surjective difference homomorphism from $\left(R^{\prime}, \sigma^{\prime}\right)$ to $(R, \sigma)$, and I a difference ideal of $R$. Then $\phi^{-1}(I)$ is proper (resp. prime, reflexive) iff I is.

Proof. Denote $\phi^{-1}(I)$ by $I^{\prime}$. Note that $I^{\prime}$ is a difference ideal. Clearly, $I^{\prime}$ is proper iff $I$ is. Assume that $I^{\prime}$ is proper. Then the induced homomorphism $\bar{\phi}$ from $R^{\prime} / I^{\prime}$ to $R / I$ is a ring isomorphism, since $\phi$ is surjective. So $I^{\prime}$ is prime iff $I$ is. Let $\bar{\sigma}^{\prime}: R^{\prime} / I^{\prime} \rightarrow R^{\prime} / I^{\prime}$ and $\bar{\sigma}: R / I \rightarrow R / I$ be the homomorphisms induced by $\sigma^{\prime}$ and $\sigma$, respectively. Then $\bar{\phi} \circ \bar{\sigma}^{\prime}=\bar{\sigma} \circ \bar{\phi}$, because $\phi \circ \sigma^{\prime}=\sigma \circ \phi$. Thus, $\bar{\sigma}^{\prime}$ is injective iff $\bar{\sigma}$ is, and $I^{\prime}$ is reflexive iff $I$ is.

For a subset $B$ of $R$, the algebraic and difference ideals generated by $B$ are denoted by $(B)$ and $[B]$, respectively. Note that $[B]$ is the algebraic ideal generated by $\cup_{\ell \in \mathbb{N}} \sigma^{\ell}(B)$. Let $I$ be a difference ideal and $H$ a subset of $R$ closed under $\sigma$ and multiplication. Then the saturation ideal

$$
I: H=\{r \in R \mid \exists h \in H, h r \in I\}
$$

is a difference ideal.

We retain the indeterminates in $\mathbf{U}$ and $\mathbf{x}$ introduced in Section 2, and let $\mathbf{X}=\mathbf{x} \cup\left\{\sigma^{\ell}\left(x_{j}\right) \mid j \in\{1, \ldots, n\}, \ell \in \mathbb{Z}^{+}\right\}$be an infinite set of indeterminates disjoint with $\mathbf{U}$. Extend $\sigma$ from $k$ to $k[\mathbf{U}, \mathbf{X}]$ by mapping $z$ to $\sigma(z)$ for all $z \in \mathbf{U} \cup \mathbf{X}$ so that $k[\mathbf{U}, \mathbf{X}]$ becomes a difference ring, which is usually denoted by $k\{\mathbf{u}, \mathbf{x}\}$. We opt for the former notation, as $k[\mathbf{U}, \mathbf{x}]$ plays a significant role in the sequel.

Definition 3.1 Given a system $\Sigma$ of the form (1), let $f_{j}=\frac{b_{j}}{a_{j}}$ with $a_{j}$ and $b_{j}$ in $k[\mathbf{U}, \mathbf{x}]$ and $\operatorname{gcd}\left(a_{j}, b_{j}\right)=1$, and let $p_{j}=a_{j} \sigma\left(x_{j}\right)-b_{j}$ for all $j$ with $1 \leq j \leq n$. Denote by $H_{\Sigma}$ the monoid generated by $\sigma^{\ell}\left(a_{j}\right)$ for all $j \in\{1, \ldots, n\}$ and $\ell \in \mathbb{N}$. We call $\left[p_{1}, \ldots, p_{n}\right]: H_{\Sigma}$ the difference ideal associated with $\Sigma$, and denote it by $I_{\Sigma}$.

The reader is referred to [8, page 74] for the definition of solutions of a difference equation. Roughly speaking, $\Sigma$ and $I_{\Sigma}$ are related as follows. A solution of $\Sigma$ is a solution of $I_{\Sigma}$. Conversely, a solution of $I_{\Sigma}$ that annihilates none of the $a_{j}$, is a solution of $\Sigma$.

We recall a reduction process for difference polynomials. The order of an element $r \in k[\mathbf{U}, \mathbf{X}]$ (with respect to $\mathbf{x}$ ) is defined to be $\ell$ if $\sigma^{\ell}(x)$ appears in $r$ for some $x \in \mathbf{x}$, and $\sigma^{e}(y)$ does not appear in $r$ for all $e>\ell$ and $y \in \mathbf{x}$. By convention, the order of any elements in $k[\mathbf{U}]$ is understood to be $-\infty$. A ranking $\prec$ is a total order on $\mathbf{X}$ such that $x \prec \sigma(x)$ for all $x \in \mathbf{X}$. A ranking is orderly if, for all $x, y$ in $\mathbf{x}, \sigma^{d}(x) \prec \sigma^{\ell}(y)$ whenever $d<\ell$.

Assume that $r$ is an element of order $\ell$ in $k[\mathbf{U}, \mathbf{X}]$. If $\ell>0$, then, using the successive difference pseudo-division by $p_{1}, \ldots, p_{n}$ with respect to an orderly ranking, we have that

$$
h r=q+r_{0}
$$

where $h$ is in $H_{\Sigma}$ with order less than $\ell, q$ is in the algebraic ideal generated in the subring $k\left[\mathbf{U}, \mathbf{x}, \sigma(\mathbf{x}), \ldots, \sigma^{\ell}(\mathbf{x})\right]$ by $\sigma^{e}\left(p_{j}\right)$ for all $e$ in $\{0,1, \ldots, \ell-1\}$ and $j$ in $\{1, \ldots, n\}$, and $r_{0}$ is in $k[\mathbf{U}, \mathbf{x}]$. If $\ell \leq 0$, we set $h=1, q=0$ and $r_{0}=r$ in (3). The reader is referred to [12] for the definition of a successive difference pseudo-division with respect to any ranking.

Example 3.2 Compute the successive difference pseudo-remainder of $r=\sigma^{2}\left(x_{1}\right)$ by $p_{1}=u \sigma\left(x_{1}\right)-x_{2}$ and $p_{2}=u \sigma\left(x_{2}\right)-x_{1}$.

Since $r$ is of order two, we first compute the pseudo-remainder of $r$ by $\sigma\left(p_{1}\right)$. This yields $\sigma(u) r=\sigma\left(p_{1}\right)+\sigma\left(x_{2}\right)$. Next, compute the pseudo-remainder of $\sigma\left(x_{2}\right)$ by $p_{2}$ to get $u \sigma\left(x_{2}\right)=p_{2}+x_{1}$. As $x_{1}$ has order zero, (3) becomes $u \sigma(u) r=u \sigma\left(p_{1}\right)+p_{2}+x_{1}$.

Most of results in this section are based on the next key lemma.

Lemma 3.2 If $I_{\Sigma}$ is proper, then $k[\mathbf{U}, \mathbf{x}] \cap I_{\Sigma}=\{0\}$.

Proof. For every $\ell \in \mathbb{N}$, we let $R^{(\ell)}=k\left[\mathbf{U}, \mathbf{x}, \sigma(\mathbf{x}), \ldots, \sigma^{\ell}(\mathbf{x})\right]$, and $H^{(\ell)}$ the monoid generated by $\sigma^{d}\left(a_{j}\right)$ for all $d \in\{0, \ldots, \ell\}$ and $j \in\{1, \ldots, n\}$. Then $H^{(\ell)}$ is contained in $R^{(\ell)}$. Put $J^{(0)}=\{0\}$ and, for all $\ell>0$, let $J^{(\ell)}$ be the algebraic ideal generated in $R^{(\ell)}$ by $\sigma^{e}\left(p_{j}\right)$ for all $e \in\{0, \ldots, \ell-1\}$ and $j \in\{1, \ldots, n\}$. Moreover, we put $I^{(0)}=\{0\}$ and, for every $\ell>0, I^{(\ell)}=J^{(\ell)}: H^{(\ell-1)}$, which is an algebraic ideal in $R^{(\ell)}$. Observe that $I_{\Sigma}=\cup_{\ell \in \mathbb{N}} I^{(\ell)}$. So it suffices to show that $R^{(0)} \cap I^{(\ell)}=\{0\}$ for all $\ell \in \mathbb{N}$.

This clearly holds for $\ell=0$. Assume now that $\ell>0$. Let $D_{\ell-1}$ be the determinant of the $n \ell \times n \ell$ matrix

$$
\frac{\partial\left(p_{1}, \ldots, p_{n}, \sigma\left(p_{1}\right), \ldots, \sigma\left(p_{n}\right), \ldots, \sigma^{\ell-1}\left(p_{1}\right), \ldots, \sigma^{\ell-1}\left(p_{n}\right)\right)}{\partial\left(\sigma\left(x_{1}\right), \ldots, \sigma\left(x_{n}\right), \sigma^{2}\left(x_{1}\right), \ldots, \sigma^{2}\left(x_{n}\right), \ldots, \sigma^{\ell}\left(x_{1}\right), \ldots, \sigma^{\ell}\left(x_{n}\right)\right)},
$$

which is in triangular form. Thus, $D_{\ell-1}$ equals the product of all the $\sigma^{e}\left(a_{j}\right)$ 's with $0 \leq e \leq \ell-1$ and $1 \leq j \leq n$. Consequently, $I^{(\ell)}$ is the ideal of $J^{(\ell)}$ saturated at $D_{\ell-1}$. It follows from Proposition 3.2 in [24] that $R^{(0)} \cap I^{(\ell)}=\{0\}$.

A longer but more elementary proof of Lemma 3.2 is given in the appendix of [17, page 56].

\section{Corollary $3.3 I_{\Sigma}$ is a prime ideal.}

Proof. It is sufficient to consider the case in which $I_{\Sigma}$ is proper. Let $r_{1}$ and $r_{2}$ be in $K[\mathbf{U}, \mathbf{X}]$ such that their product belongs to $I_{\Sigma}$. By (3), $h_{i} r_{i} \equiv r_{i}^{\prime} \bmod I_{\Sigma}$ for some $h_{i}$ in $H_{\Sigma}$ and $r_{i}^{\prime}$ in $k[\mathbf{U}, \mathbf{x}], i=1,2$. Then $r_{1}^{\prime} r_{2}^{\prime}$ is in $I_{\Sigma}$. By Lemma 3.2, $r_{1}^{\prime} r_{2}^{\prime}=0$, which implies that either $r_{1}$ or $r_{2}$ is in $I_{\Sigma}$.

The ideal $I_{\Sigma}$ is not always proper, that is, $\Sigma$ may be inconsistent; and $I_{\Sigma}$ is not necessarily reflexive, that is, there may exist a nonzero element in $k[\mathbf{U}, \mathbf{x}] \backslash I_{\Sigma}$ vanishing at any solution of $\Sigma$.

Example 3.3 (i) Let $\Sigma=\left\{\sigma\left(x_{1}\right)=x_{1}, \sigma\left(x_{2}\right)=x_{1}, \sigma\left(x_{3}\right)=\frac{u_{1}}{x_{2}-x_{1}}\right\}$. Then $p_{1}=\sigma\left(x_{1}\right)-x_{1}, p_{2}=\sigma\left(x_{2}\right)-x_{1}$ and $p_{3}=a_{3} \sigma\left(x_{3}\right)-u_{1}$. with $a_{3}=x_{2}-x_{1}$. Since $\sigma\left(a_{3}\right)=p_{2}-p_{1}, \sigma\left(a_{3}\right) \in\left[p_{1}, p_{2}, p_{3}\right] \cap H_{\Sigma}$. So $I_{\Sigma}$ is improper, that is, $\Sigma$ has no solution in any difference field.

(ii) Let $\Sigma=\left\{\sigma\left(x_{1}\right)=x_{2}, \sigma\left(x_{2}\right)=x_{2}\right\}$. Then $\sigma\left(x_{1}\right)=\sigma\left(x_{2}\right)$. Hence, $x_{1}-x_{2}$ vanishes at every solution of $\Sigma$, but it is not in $I_{\Sigma}$ as will be shown in Lemma 3.2. So $I_{\Sigma}$ is not reflexive.

Note that neither of the systems in Example 3.3 is submersive.

Proposition 3.4 Let $\Sigma$ be a rational difference system given by (1). Then $I_{\Sigma}$ is proper, prime and reflexive iff $\Sigma$ is submersive. When 
this is the case, $(k(\mathbf{U}, \mathbf{x}), \theta)$ is $k$-isomorphic to $\left(K_{\Sigma}, \bar{\sigma}\right)$, where $\theta$ is defined in Proposition 2.4, $K_{\Sigma}$ is the quotient field of $k[\mathbf{U}, \mathbf{X}] / I_{\Sigma}$, and $\bar{\sigma}$ is induced by $\sigma$.

Proof. Assume that $\Sigma$ is submersive. Then $(k(\mathbf{U}, \mathbf{x}), \theta)$ is a welldefined difference field by Proposition 2.4. Define $\phi$ to be a difference homomorphism from $(k[\mathbf{U}, \mathbf{X}], \sigma)$ to $(k(\mathbf{U}, \mathbf{x}), \theta)$ that fixes $k$ and maps $z$ to $z$ for all $z \in \mathbf{U} \cup \mathbf{x}$. Then

$$
\phi\left(\sigma\left(x_{j}\right)\right)=\theta\left(\phi\left(x_{j}\right)\right)=\theta\left(x_{j}\right)=f_{j} \quad \text { for all } j \text { with } 1 \leq j \leq n .
$$

Thus, $\left[p_{1}, \ldots, p_{n}\right]$ is a subset of $\operatorname{ker}(\phi)$. Moreover, $H_{\Sigma}$ and $\operatorname{ker}(\phi)$ are disjoint, since $\phi\left(\sigma^{\ell}\left(a_{j}\right)\right)=\theta^{\ell}\left(\phi\left(a_{j}\right)\right) \neq 0$. It follows that $I_{\Sigma}$ is contained in $\operatorname{ker}(\phi)$. To show $\operatorname{ker}(\phi) \subset I_{\Sigma}$, assume $r \in \operatorname{ker}(\phi)$. By (3), $h r \equiv r_{0} \bmod \left[p_{1}, \ldots, p_{n}\right]$ for some $h \in H_{\Sigma}$ and $r_{0} \in k[\mathbf{U}, \mathbf{x}]$. Thus, $\phi\left(r_{0}\right)=0$, and so $r_{0}=0$ by the definition of $\phi$. We have that $r \in I_{\Sigma}$. Consequently, $I_{\Sigma}=\operatorname{ker}(\phi)$, which, together with Theorem $\mathrm{V}$ in [8, page 71], implies that $I_{\Sigma}$ is proper, prime and reflexive.

Conversely, assume that $I_{\Sigma}$ is proper, prime and reflexive. It suffices to show that $\sigma(\mathbf{U}) \cup\left\{f_{1}, \ldots, f_{n}\right\}$ is algebraically independent over $k$ by Lemma 2.3. Let $p$ be a polynomial over $k$ such that

$$
p\left(\sigma(U), f_{1}, \ldots, f_{n}\right)=0 .
$$

Denote $p\left(\sigma(U), \sigma\left(x_{1}\right), \ldots, \sigma\left(x_{n}\right)\right)$ by $r$, which is an element of order less than two in $k[\sigma(U), \sigma(\mathbf{x})]$. By (3), $h r \equiv r_{0} \bmod \left(p_{1}, \ldots, p_{n}\right)$ for some $h$ in $H_{\Sigma} \cap k[\mathbf{U}, \mathbf{x}]$ and $r_{0}$ in $k[\mathbf{U}, \mathbf{x}]$. Replacing $\sigma\left(x_{j}\right)$ by $f_{j}$, $j=1, \ldots, n$, in the above congruence, we see that $r_{0}=0$ by (4). Consequently, $r$ belongs to $I_{\Sigma}$. Since $\sigma$ is an automorphism of $k$, there exists $r^{\prime}$ in $k[\mathbf{U}, \mathbf{x}]$ such that $\sigma\left(r^{\prime}\right)=r$. So $r^{\prime}$ is in $I_{\Sigma}$, since $I_{\Sigma}$ is reflexive. By Lemma 3.2, $r^{\prime}$ is zero, so is $r$, and, hence, $p$ is zero. The set $\sigma(\mathbf{U}) \cup\left\{f_{1}, \ldots, f_{n}\right\}$ is algebraically independent over $k$.

Finally, assume that $\Sigma$ is submersive. Then the homomorphism $\phi$ defined in the first paragraph of the proof induces a difference isomorphism from $\left(K_{\Sigma}, \overline{\boldsymbol{\sigma}}\right)$ to $(k(\mathbf{U}, \mathbf{x}), \theta)$.

For a submersive system $\Sigma,\left(K_{\Sigma}, \bar{\sigma}\right)$ is the difference field generated by $\mathbf{z}=\left(u_{1}+I_{\Sigma}, \ldots, u_{m}+I_{\Sigma}, x_{1}+I_{\Sigma}, \ldots, x_{n}+I_{\Sigma}\right)$, which is a generic zero of $I_{\Sigma}$ ([8, page 76]). The vector $\mathbf{z}$ is also a solution of $\Sigma$, because any $a_{j}$ does not vanish at $\mathbf{z}$ by Lemma 3.2. So we call $\left(K_{\Sigma}, \bar{\sigma}\right)$ the difference field associated with $\Sigma$. Note that $\left(K_{\Sigma}, \bar{\sigma}\right)$ is not inversive if $\mathbf{u}$ is nonempty. In the next section, we will construct the inversive closure of $\left(K_{\Sigma}, \bar{\sigma}\right)$.

Remark 3.4 For a submersive system $\Sigma$, there are two ways to determine the membership of $I_{\Sigma}$. One is to use successive pseudodivision, i.e. $r \in I_{\Sigma}$ iff $r_{0}=0$ by (3). The other is to use $\phi$ defined in the proof of Proposition 3.4, i.e. $r \in I_{\Sigma}$ iff $\phi(r)=0$. The latter also yields a rational normal form function from $k[\mathbf{U}, \mathbf{X}] / I_{\Sigma}$ to $k(\mathbf{U}, \mathbf{x})$.

\section{INVERSIVE CLOSURES}

Let $J$ be a proper, prime and reflexive ideal in $k[\mathbf{U}, \mathbf{X}], K$ the quotient field of $k[\mathbf{U}, \mathbf{X}] / J$, and $\bar{\sigma}: K \rightarrow K$ the monomorphism induced by $\sigma$. In this section, we construct the inversive closure $(\widehat{K}, \hat{\sigma})$ of $(K, \bar{\sigma})$, which is an inversive field extension of $K$ with the property that, for every $\hat{f} \in \widehat{K}$, there exists $\ell \in \mathbb{N}$ such that $\hat{\sigma}^{\ell}(\hat{f})$ belongs to $K$ (see [8, page 66]). This inversive field will serve as a base field to analyze discrete-time systems in Section 7, when $J$ is the ideal associated with a submersive system (equation).

Initially, we denote $k[\mathbf{U}, \mathbf{X}]$ by $R_{0}, u_{i}$ by $u_{i, 0}$, and $x_{j}$ by $x_{j, 0}$ for all $i, j$ with $1 \leq i \leq m$ and $1 \leq j \leq n$. For every $\ell \in \mathbb{Z}^{+}$, we set $R_{\ell}=R_{\ell-1}\left[u_{1,-\ell}, \ldots, u_{m,-\ell}, x_{1,-\ell}, \ldots, x_{n,-\ell}\right]$ inductively. Extend $\sigma$ from $R_{\ell-1}$ to $R_{\ell}$ by mapping $u_{i,-\ell}$ to $u_{i,-(\ell-1)}$ and $x_{j,-\ell}$ to $x_{j,-(\ell-1)}$, where $i=1, \ldots, m$ and $j=1, \ldots, n$. This process yields an ascending chain: $R_{0} \subset R_{1} \subset \cdots \subset R_{\ell} \subset \cdots$ of difference rings, in which $\sigma\left(R_{\ell}\right)=R_{\ell-1}$.
Denote $J$ by $J_{0}$, which is in $R_{0}$. Since $\sigma\left(R_{1}\right)=R_{0}, \sigma^{-1}\left(J_{0}\right)$ is well-defined in $R_{1}$. We set $J_{1}=\sigma^{-1}\left(J_{0}\right)$. Define $J_{\ell}=\sigma^{-1}\left(J_{\ell-1}\right)$ for all $\ell \in \mathbb{Z}^{+}$recursively.

Lemma 4.1 For every $\ell \in \mathbb{Z}^{+}, J_{\ell} \cap R_{\ell-1}=J_{\ell-1}$, and, moreover, $J_{\ell}$ is proper, prime and reflexive.

Proof. Since $\sigma\left(J_{0}\right) \subset J_{0}, J_{0} \subset J_{1}$ by definition. So $J_{0} \subset J_{1} \cap R_{0}$. For $r \in J_{1} \cap R_{0}, \sigma(r) \in J_{0}$. Hence, $r$ belongs to $J_{0}$, since $J_{0}$ is reflexive. This proves $J_{0}=R_{0} \cap J_{1}$. The ideal $J_{1}$ is proper, prime and reflexive by Lemma 3.1. If $J_{\ell}$ has the properties given in the statement of this lemma, so does $J_{\ell+1}$ by the same argument, in which $J_{0}$ is replaced by $J_{\ell}$, and $J_{1}$ by $J_{\ell+1}$.

By Lemma 4.1, we have an ascending chain $J_{0} \subset J_{1} \subset \cdots \subset J_{\ell} \subset$ $\cdots$, in which each $J_{\ell}$ is a proper, prime and reflexive ideal in $R_{\ell}$, moreover, $J_{\ell+1}=\sigma^{-1}\left(J_{\ell}\right)$ and $J_{\ell+1} \cap R_{\ell}=J_{\ell}$.

The two chains allow us to form a ring $\widehat{R}=\cup_{\ell \in \mathbb{N}} R_{\ell}$ and an ideal $\widehat{J}=\cup_{\ell \in \mathbb{N}} J_{\ell}$, respectively. Extend $\sigma$ to a map from $\widehat{R}$ to itself by mapping $r$ to $\sigma(r)$ if $r \in R_{\ell}$ for some $\ell \in \mathbb{N}$. It is direct to verify that $\sigma$ is a well-defined automorphism of $\widehat{R}$, and, therefore, $(\widehat{R}, \sigma)$ is an inversive difference ring. By Lemma 4.1, $J_{\ell}=\widehat{J} \cap R_{\ell}$ for all $\ell \in \mathbb{N}$, and $\widehat{J}$ is a proper, prime and reflexive ideal.

Proposition 4.2 With the notation just introduced, let $(\widehat{R} / \widehat{J}, \hat{\sigma})$ be the induced ring, and $(\widehat{K}, \hat{\sigma})$ be its fraction field. Then $(\widehat{K}, \hat{\sigma})$ is the inversive closure of $(K, \bar{\sigma})$.

Proof. The ring $(\widehat{R} / \widehat{J}, \hat{\sigma})$ is inversive, because $(\widehat{R}, \sigma)$ is inversive. Consequently, $(\widehat{K}, \hat{\sigma})$ is an inversive field.

Let $\tau_{0}: R_{0} \rightarrow \widehat{R} / \widehat{J}$ be defined by $r \mapsto r+\widehat{J}$. Then $\operatorname{ker}\left(\tau_{0}\right)$ is equal to $J_{0}$ by Lemma 4.1. Hence, $\tau:\left(R_{0} / J_{0}, \bar{\sigma}\right) \rightarrow(\widehat{R} / \widehat{J}, \hat{\sigma})$ defined by $r+J_{0} \mapsto r+\widehat{J}$ for all $r \in R_{0}$, is a difference monomorphism. So $\tau$ can be extended to a difference monomorphism from $K$ to $\widehat{K}$.

For every $r \in \widehat{K}$, there exist $\ell \in \mathbb{N}$ and $p_{\ell}, q_{\ell} \in R_{\ell}$ such that $\hat{r}$ equals $\frac{p_{\ell}+\widehat{J}}{q_{\ell}+\widehat{J}}$. Thus, $\hat{\sigma}^{\ell}(\hat{r})$ belongs to $\tau(K)$. By Theorem II in [8, page 66], $\widehat{K}$ is the inversive closure of $\tau(K)$, and so is that of $K$. $\square$

Assume now that $J$ is a difference ideal associated with a submersive system $\Sigma$ given by (1). By Propositions 3.4 and 4.2, the field $K_{\Sigma}$ associated with $\Sigma$ has an inversive closure, which is called the inversive field associated with $\Sigma$, and is denoted by $\left(\widehat{K}_{\Sigma}, \hat{\sigma}\right)$.

The field $\widehat{K}_{\Sigma}$ may also be understood as the direct limit of the quotient fields of $R_{\ell} / J_{\ell}$ for all $\ell \in \mathbb{N}$, each of which is isomorphic to a field of rational functions by Proposition 3.4.

There are two ways to determine, for $r \in \widehat{R}$, whether $r+\widehat{J}$ is equal to zero in $\widehat{K}_{\Sigma}$. Assume $r \in R_{\ell}$ for some $\ell \in \mathbb{N}$. First, Lemma 4.1 implies $r \in \widehat{J}$ iff $r \in J_{\ell}$. The latter is equivalent to that $\sigma^{\ell}(r)$ is in $J$, which can be determined by the methods described in Remark 3.4. Second, note that $J_{\ell}=\left[\sigma^{-\ell}\left(p_{1}\right), \ldots, \sigma^{-\ell}\left(p_{n}\right)\right]: \sigma^{-\ell}\left(H_{\Sigma}\right)$. Then both Lemma 3.2 and Proposition 3.4 hold for $J_{\ell}$ when we replace $\mathbf{u}$ by $\sigma^{-\ell}(\mathbf{u})$, and $\mathbf{x}$ by $\sigma^{-\ell}(\mathbf{x})$. So the methods in Remark 3.4 are applicable in the membership problem of $J_{\ell}$.

Example 4.1 Let $\Sigma$ be $\left\{\sigma\left(x_{1}\right)=\frac{x_{2}}{u}, \sigma\left(x_{2}\right)=\frac{x_{1}^{2}}{u}\right\}$, which is a submersive system. Let $p_{1}=u \sigma\left(x_{1}\right)-x_{2}$ and $p_{2}=u \sigma\left(x_{2}\right)-x_{1}^{2}$, and let $H_{\Sigma}$ be the monoid generated by $u, \sigma(u), \ldots$ Then the ideal associated with $\Sigma$ is $I_{\Sigma}=\left[p_{1}, p_{2}\right]: H_{\Sigma}$. Let $r=u_{-1} u_{-2} x_{1}-x_{1,-2}^{2}$ be in $\widehat{R}$. We determine whether $r+\widehat{I}_{\Sigma}$ is zero in the inversive field associated with $\Sigma$. It suffices to determine whether $\sigma^{2}(r)$ is in $I_{\Sigma}$. By (3), we have that $u \sigma(u) \sigma^{2}(r)=u^{2} \sigma(u) \sigma\left(p_{1}\right)+u \sigma(u) p_{2}$. Hence $\sigma^{2}(r)$ is in $I_{\Sigma}$, and, consequently, $r+\widehat{I}_{\Sigma}$ is zero. 


\section{KÄHLER DIFFERENTIALS AND TOR- SION ELEMENTS}

We show that Kähler differentials of the inversive field associated with a submersive system form a module over a ring of skew polynomials, and determine its torsion submodule. Later, in Section 7, we point out that a submersive rational discrete-time system is accessible iff the corresponding module is torsion-free. This is in agreement with results known for nonlinear continuous-time systems [10], linear systems over Ore algebras [6], and linear timedelay systems [11].

In this section, all modules and linear spaces are left ones. Let $\Sigma$ be a submersive system given by (1) whose associated difference ideal and inversive field are denoted by $I_{\Sigma}$ and $\left(\widehat{K}_{\Sigma}, \hat{\sigma}\right)$, respectively. Let $(k(\mathbf{U}, \mathbf{x}), \theta)$ and $\left(K_{\Sigma}, \bar{\sigma}\right)$ be the same as those in Proposition 3.4. Define $\psi$ to be the $k$-homomorphism from $k(\mathbf{U}, \mathbf{x})$ to $K_{\Sigma}$ that maps $z$ to $z+I_{\Sigma}$ for all $z \in \mathbf{U} \cup \mathbf{x}$. Then $\psi$ is a difference $k$ isomorphism by the proof of Proposition 3.4. So $\psi$ induces a difference $k$-monomorphism $\widehat{\psi}$ from $k(\mathbf{U}, \mathbf{x})$ to $\widehat{K}_{\Sigma}$ that maps $z$ to $z+\widehat{I}_{\Sigma}$ for all $z \in \mathbf{U} \cup \mathbf{x}$. This observation allows us to abridge $z+\widehat{I}_{\Sigma}$ in $\widehat{K}_{\Sigma}$ as $z$ for all $z \in \mathbf{U} \cup \mathbf{x}$. Hence, we have, in $\widehat{K}_{\Sigma}$,

$$
\hat{\sigma}\left(x_{j}\right)=f_{j}, \quad j=1, \ldots, n .
$$

Let $S_{\Sigma}=\widehat{K}_{\Sigma}[s ; \hat{\sigma}]$ be the ring of $\hat{\sigma}$-shift operators over $\widehat{K}_{\Sigma}$, which is a special case for Ore algebras [27, 7, 4]. The commutation rule of $S_{\Sigma}$ is $s r=\hat{\sigma}(r) s$ for all $r \in \widehat{K}_{\Sigma}$.

Let $\Omega_{\Sigma}$ be the vector space spanned by $k$-differentials over $\widehat{K}_{\Sigma}$. We view $\Omega_{\Sigma}$ as a module over $S_{\Sigma}$ in the following manner. By Lemma 9.1.2 in [3], the automorphism $\hat{\sigma}$ of $\widehat{K}_{\Sigma}$ induces a unique skew-linear map $\sigma^{*}$ from $\Omega_{\Sigma}$ to itself, that is, $\sigma^{*}$ is additive, and $\sigma^{*}(r \omega)=\hat{\sigma}(r) \sigma^{*}(\omega)$ for all $r \in \widehat{K}_{\Sigma}$ and $\omega \in \Omega_{\Sigma}$. Moreover, the diagram

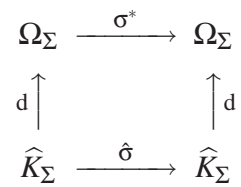

is commutative. Define $s \omega$ to be $\sigma^{*}(\omega)$ for all $\omega$ in $\Omega_{\Sigma}$. The skewlinearity of $\sigma^{*}$ implies that $s(r \omega)=\hat{\sigma}(r)(s \omega)$ for all $r$ in $K_{\Sigma}$ and $\omega$ in $\Omega_{\Sigma}$. Then $\Omega_{\Sigma}$ becomes a left module over $S_{\Sigma}$. Moreover, the commutativity of (6) implies $s(g \mathrm{~d} h)=\hat{\sigma}(g) \mathrm{d} \hat{\sigma}(h)$ for all $g, h$ in $\widehat{K}_{\Sigma}$.

An element $\omega$ of $\Omega_{\Sigma}$ is a torsion element if $\omega$ is annihilated by a nonzero polynomial in $S_{\Sigma}$, that is, the submodule generated by $\omega$, denoted by $S_{\Sigma} \omega$, is a finite-dimensional linear subspace over $\widehat{K}_{\Sigma}$. The set of all torsion elements in $\Omega_{\Sigma}$ is denoted by $\operatorname{tor}\left(\Omega_{\Sigma}\right)$. The existence of the least common left multiples in $S_{\Sigma}$ implies that tor $\left(\Omega_{\Sigma}\right)$ is a submodule [7, page 46]. The module $\Omega_{\Sigma}$ is said to be torsionfree if $\operatorname{tor}\left(\Omega_{\Sigma}\right)$ equals $\{0\}$.

Let $\Omega_{0}=\operatorname{span}_{\widehat{K}_{\Sigma}}\{\mathrm{d} z \mid z \in \mathbf{U} \cup \mathbf{x}\}$. Differentiating (5), we see that $s \mathrm{~d} z$ belongs to $\Omega_{0}$ for all $z \in \mathbf{U} \cup \mathbf{x}$. So $\Omega_{0}$ is a submodule of $\Omega_{\Sigma}$. The next lemma will help us shift an element in $\Omega_{\Sigma}$ to $\Omega_{0}$, and asserts that $\{\mathrm{d} z \mid z \in \mathbf{U} \cup \mathbf{x}\}$ is a $\widehat{K}_{\Sigma}$-basis of $\Omega_{0}$.

Lemma 5.1 (i) If $\omega$ is a nonzero element in $\Omega_{\Sigma}$, then $s \omega$ is nonzero. (ii) In $\Omega_{\Sigma},\{\mathrm{d} z \mid z \in \mathbf{U} \cup \mathbf{x}\}$ is linearly independent over $\widehat{K}_{\Sigma}$.

Proof. (i) Let $\omega=\sum_{i} g_{i} \mathrm{~d} h_{i}$, where $g_{i}, h_{i} \in \widehat{K}_{\Sigma}$, and the set $\left\{\mathrm{d} h_{i}\right\}$ is linearly independent over $\widehat{K}_{\Sigma}$. Suppose that $s \omega=0$. Then $\left\{\mathrm{d} \hat{\sigma}\left(h_{i}\right)\right\}$ is linearly dependent over $\widehat{K}_{\Sigma}$, since $s \omega=\sum_{i} \hat{\sigma}\left(g_{i}\right) \mathrm{d} \hat{\sigma}\left(h_{i}\right)$. It follows from Lemma 2.1 that $\left\{\hat{\sigma}\left(h_{i}\right)\right\}$ is algebraically dependent over $k$, and so is $\left\{h_{i}\right\}$, because $\hat{\sigma}$ extends $\sigma$, which is an automorphism. So $\left\{\mathrm{d} h_{i}\right\}$ is linearly dependent over $\widehat{K}_{\Sigma}$, a contradiction. (ii) $\mathbf{U} \cup \mathbf{x}$, viewed as a subset of $k(\mathbf{U}, \mathbf{x})$, is algebraically independent over $k$. So the assertion follows from the definition of $\widehat{\psi}$ given above and Lemma 2.1.

We make an additional assumption in the rest of this section that $f_{1}, \ldots, f_{n}$ in (1) are in $k(\mathbf{u}, \mathbf{x})$ instead of $k(\mathbf{U}, \mathbf{x})$. Though this assumption helps to simplify the calculations in $\Omega_{\Sigma}$, it is not restrictive and the same results can be derived in the general case. Denote the column vectors $\left(u_{1}, \ldots, u_{m}\right)^{T}$ and $\left(x_{1}, \ldots, x_{n}\right)^{T}$ by $\vec{u}$ and $\vec{x}$, respectively. Differentiating (5) yields

$$
s \mathrm{~d} \vec{x}=A \mathrm{~d} \vec{x}+B \mathrm{~d} \vec{u},
$$

where $A=\frac{\partial\left(f_{1}, \ldots, f_{n}\right)}{\partial\left(x_{1}, \ldots, x_{n}\right)}$ and $B=\frac{\partial\left(f_{1}, \ldots, f_{n}\right)}{\partial\left(u_{1}, \ldots, u_{m}\right)}$.

We use the method described in [2] to determine tor $\left(\Omega_{\Sigma}\right)$. Let $\mathcal{H}_{1}$ be the linear subspace spanned by $\mathrm{d} x_{1}, \ldots, \mathrm{d} x_{n}$ over $\widehat{K}_{\Sigma}$, and define $\mathcal{H}_{\ell+1}$ to be $\left\{\omega \in \mathcal{H}_{\ell} \mid s \omega \in \mathcal{H}_{\ell}\right\}$ for all $\ell \in \mathbb{N}$. Then

$$
\mathcal{H}_{1} \supseteq \mathcal{H}_{2} \supseteq \cdots
$$

is a chain of finite-dimensional linear subspaces over $\widehat{K}_{\Sigma}$. Moreover, we let $\mathcal{H}_{\infty}=\cap_{\ell \in \mathbb{Z}^{+}} \mathcal{H}_{\ell}$. Since $s \mathcal{H}_{\infty} \subset \mathcal{H}_{\infty}, \mathcal{H}_{\infty}$ is a submodule.

Lemma 5.2 and Proposition 5.3 below show how the torsion submodule of $\Omega_{\Sigma}$ is related to $\mathcal{H}_{\infty}$, the submodule of autonomous oneforms described in [2] and Section 7.

Lemma 5.2 $\mathcal{H}_{\infty}=\operatorname{tor}\left(\Omega_{\Sigma}\right) \cap \Omega_{0}$.

Proof. We claim that $\operatorname{tor}\left(\Omega_{\Sigma}\right) \cap \Omega_{0} \subset \mathcal{H}_{1}$. Assume that $\omega$ is in $\Omega_{0}$. Then $\omega=\sum_{j=1}^{n} g_{j} \mathrm{~d} x_{j}+\sum_{i=1}^{m} P_{i} \mathrm{~d} u_{i}$ for some $g_{j}$ in $\widehat{K}_{\Sigma}$ and $P_{i}$ in $S_{\Sigma}$, because $\mathrm{d} \hat{\sigma}^{\ell}\left(u_{i}\right)=s^{\ell} \mathrm{d} u_{i}$ for all $\ell \in \mathbb{N}$. Suppose that $\omega$ is not in $\mathcal{H}_{1}$. Then, without loss of generality, we assume that $P_{1}$ has a nonzero leading term $c_{1} s^{d_{1}}$, where $c_{1}$ is in $\widehat{K}_{\Sigma}$ and $d_{1}$ in $\mathbb{N}$. Expanding $s^{\ell} \omega$ as a $\widehat{K}_{\Sigma}$-linear combination of $\{\mathrm{d} z \mid z \in \mathbf{U} \cup \mathbf{x}\}$, we see that $s^{\ell} \omega$ involves the term $\hat{\sigma}^{\ell}\left(c_{1}\right) \mathrm{d} \sigma^{d_{1}+\ell}\left(u_{1}\right)$ but is free of any $\mathrm{d} \sigma^{e}\left(u_{1}\right)$, where $e$ is greater than $d_{1}+\ell$. Hence, $\left\{s^{\ell} \omega \mid \ell \in \mathbb{N}\right\}$ is linearly independent over $\widehat{K}_{\Sigma}$ by Lemma 5.1 (ii). So $\omega$ is not a torsion element. The claim is proved.

Let $\omega$ be an element of the submodule $\operatorname{tor}\left(\Omega_{\Sigma}\right) \cap \Omega_{0}$. Then $S_{\Sigma} \omega$ is a subset of $\operatorname{tor}\left(\Omega_{\Sigma}\right) \cap \Omega_{0}$. By the claim, $S_{\Sigma} \omega$ is a subset of $\mathcal{H}_{1}$. By the definition of $\mathcal{H}_{\ell}, S_{\Sigma} \omega$ is contained in $\mathcal{H}_{\ell}$ for all $\ell \in \mathbb{Z}^{+}$. It follows that $\omega \in \mathcal{H}_{\infty}$. Hence, $\operatorname{tor}\left(\Omega_{\Sigma}\right) \cap \Omega_{0} \subset \mathcal{H}_{\infty}$. On the other hand, every element of $\mathcal{H}_{\infty}$ is a torsion element, because $\mathcal{H}_{\infty}$ is both a submodule over $S_{\Sigma}$ and a finite-dimensional linear subspace over $\widehat{K}_{\Sigma}$. So $\mathcal{H}_{\infty} \subset \operatorname{tor}\left(\Omega_{\Sigma}\right) \cap \Omega_{0}$.

The next proposition describes $\operatorname{tor}\left(\Omega_{\Sigma}\right)$ by $\mathcal{H}_{\infty}$.

Proposition 5.3 (i) $\operatorname{tor}\left(\Omega_{\Sigma}\right)=\left\{\omega \in \Omega_{\Sigma} \mid s^{\ell} \omega \in \mathcal{H}_{\infty}\right.$ for some $\left.\ell \in \mathbb{N}\right\}$. (ii) $\Omega_{\Sigma}$ is torsion-free iff $\mathcal{H}_{\infty}$ is trivial.

Proof. (i) Assume that $\omega$ is in $\operatorname{tor}\left(\Omega_{\Sigma}\right)$. Then there exists a positive integer $\ell$ such that $s^{\ell} \omega$ belongs to $\Omega_{0}$ by (7) and the definition of scalar multiplication. Hence, $s^{\ell} \omega$ is in $\operatorname{tor}\left(\Omega_{\Sigma}\right) \cap \Omega_{0}$, and so it is in $\mathcal{H}_{\infty}$ by Lemma 5.2. Conversely, let $\omega$ be in $\Omega_{\Sigma}$ such that $s^{\ell} \omega$ is in $\mathcal{H}_{\infty}$ for some $\ell \in \mathbb{N}$. Then $s^{\ell} \omega$ is in $\operatorname{tor}\left(\Omega_{\Sigma}\right)$ by Lemma 5.2, and so is $\omega$ by the definition of torsion elements.

(ii) It suffices to show that $\mathcal{H}_{\infty}=\{0\}$ implies tor $\left(\Omega_{\Sigma}\right)=\{0\}$ by Lemma 5.2. For $\omega \in \operatorname{tor}\left(\Omega_{\Sigma}\right)$, there exists $\ell \in \mathbb{N}$ such that $s^{\ell} \omega=0$ by the first assertion and $\mathcal{H}_{\infty}=\{0\}$. So $\omega=0$ by Lemma 5.1 (i). $\square$

To determine a $\widehat{K}_{\Sigma}$-basis of $\mathcal{H}_{\infty}$, we describe $\mathcal{H}_{\infty}$ without using any recursion.

Proposition $5.4 \mathcal{H}_{\infty}=\left\{\omega \in \mathcal{H}_{1} \mid s^{n} \omega \in \mathcal{H}_{1}\right\}$, where $n$ is the number of elements in $\mathbf{x}$ (also known as the order of the system $\Sigma$ ). 
Proof. By the definition of chain (8), we conclude that $\mathcal{H}_{i}=\mathcal{H}_{\infty}$ if $\mathcal{H}_{i}=\mathcal{H}_{i+1}$, and that $\operatorname{dim}_{\widehat{K}_{\Sigma}} \mathcal{H}_{i}<\operatorname{dim}_{\widehat{K}_{\Sigma}} \mathcal{H}_{i+1}$ if $\mathcal{H}_{i+1} \varsubsetneqq \mathcal{H}_{i}$. Therefore, $\mathcal{H}_{n+1}=\mathcal{H}_{\infty}$. It remains to show $\mathcal{H}_{n+1}=\left\{\omega \in \mathcal{H}_{1} \mid s^{n} \omega \in \mathcal{H}_{1}\right\}$. We shall prove that, for all $\ell \in \mathbb{Z}^{+}, \mathcal{H}_{\ell}=\mathcal{H}_{\ell}^{\prime}$, where $\mathcal{H}_{\ell}^{\prime}$ stands for $\left\{\omega \in \mathcal{H}_{1} \mid s^{\ell-1} \omega \in \mathcal{H}_{1}\right\}$. To this end we need:

Claim. Let $\omega$ be an element of $\Omega_{\Sigma}$ and $\ell$ be a positive integer. If both $\omega$ and $s^{\ell} \omega$ are in $\mathcal{H}_{1}$, then $s^{i} \omega$ is in $\mathcal{H}_{1}$ for all $i=0, \ldots, \ell-1$.

The claim clearly holds for $i=0$. Assume $s^{i-1} \omega \in \mathcal{H}_{1}$ for some $i$ with $0<i<\ell$. By (7) and Lemma 5.1, $s^{i} \omega$ can be uniquely written as $\omega_{0}+\omega_{1}$ for some $\omega_{0} \in \mathcal{H}_{1}$ and $\omega_{1} \in \operatorname{span}_{\widehat{K}_{\Sigma}}\left\{\mathrm{d} u_{1}, \ldots, \mathrm{d} u_{m}\right\}$. Thus, $s^{\ell} \omega=s^{\ell-i} s^{i} \omega=s^{\ell-i} \omega_{0}+s^{\ell-i} \omega_{1}$. Observe that $s^{\ell-i} \omega_{0}$ is free of $\mathrm{d} \hat{\sigma}^{\ell-i}\left(u_{1}\right), \ldots, \mathrm{d} \hat{\sigma}^{\ell-i}\left(u_{m}\right)$, but $s^{\ell-i} \omega_{1}$ is a $\widehat{K}_{\Sigma}$-linear combination of $\mathrm{d} \hat{\sigma}^{\ell-i}\left(u_{1}\right), \ldots, \mathrm{d} \hat{\sigma}^{\ell-i}\left(u_{m}\right)$. It follows from $s^{\ell} \omega \in \mathcal{H}_{1}$ that $s^{\ell-i} \omega_{1}=0$. By Lemma 5.1 (i), $\omega_{1}=0$. The claim is proved.

Clearly, $\mathcal{H}_{1}=\mathcal{H}_{1}^{\prime}$. Assume that $\mathcal{H}_{\ell-1}=\mathcal{H}_{\ell-1}^{\prime}$. If $\omega$ is in $\mathcal{H}_{\ell}$, then $s \omega$ is $\mathcal{H}_{\ell-1}$, and so $s \omega$ is in $\mathcal{H}_{\ell-1}^{\prime}$. Hence, $s^{\ell-1} \omega$ is in $\mathcal{H}_{1}$. Consequently, $\omega$ is in $\mathcal{H}_{\ell}^{\prime}$. Conversely, assume that $\omega$ belongs to $\mathcal{H}_{\ell}^{\prime}$. Then both $s \omega$ and $s^{\ell-2} \omega$ are in $\mathcal{H}_{1}$ by the claim. Then $\omega \in \mathcal{H}_{\ell-1}^{\prime}$ since $\omega, s^{\ell-2} \omega \in \mathcal{H}_{1}$. Likewise, $s \omega \in \mathcal{H}_{\ell-1}^{\prime}$ since $s \omega, s^{\ell-1} \omega \in \mathcal{H}_{1}$. So $\omega \in \mathcal{H}_{\ell}$, because $\omega, s \omega \in \mathcal{H}_{\ell-1}$ by the induction hypothesis. $\square$

A $\widehat{K}_{\Sigma}$-basis of $\mathcal{H}_{\infty}$ can be determined by computing the left kernel of an $n \times m n$ matrix over $\widehat{K}_{\Sigma}$, as stated in the next proposition.

Proposition 5.5 Let $A$ and $B$ be given by (7). Define $C_{1}=\hat{\sigma}^{-1}(B)$ and $C_{i+1}=\hat{\sigma}^{-1}(A) \hat{\sigma}^{-1}\left(C_{i}\right)$ for all $i \in \mathbb{Z}^{+}$, and define $C$ to be the $n \times$ mn matrix $\left(C_{1}, \ldots, C_{n}\right)$. Let $\omega=\left(h_{1}, \ldots, h_{n}\right) \mathrm{d} \vec{x}$. Then $\omega$ is in $\mathcal{H}_{\infty}$ iff $\left(h_{1}, \ldots, h_{n}\right)$ is in the left kernel of $C$.

Proof. By Proposition 5.4, $\omega$ belongs to $\mathcal{H}_{\infty}$ iff $s^{n} \omega$ is in $\mathcal{H}_{1}$. Let $A_{0}$ be the $n \times n$ identity matrix, and define $A_{\ell}=\hat{\sigma}\left(A_{\ell-1}\right) A$ for all $\ell \in \mathbb{Z}^{+}$. An easy induction shows that

$$
A_{\ell}=\hat{\sigma}^{\ell-1}(A) A_{\ell-1} \quad \text { and } \quad \hat{\sigma}^{\ell}\left(C_{\ell}\right)=\hat{\sigma}\left(A_{\ell-1}\right) B,
$$

which, together with (7), imply $s^{\ell} \mathrm{d} \vec{x}=A_{\ell} \mathrm{d} \vec{x}+\sum_{i=1}^{\ell} \hat{\sigma}^{\ell}\left(C_{i}\right) \mathrm{d} \hat{\sigma}^{\ell-i}(\vec{u})$ for all $\ell \in \mathbb{Z}^{+}$. So $s^{n} \omega$ is in $\mathcal{H}_{1}$ iff $\left(\hat{\sigma}^{n}\left(h_{1}\right), \ldots, \hat{\sigma}^{n}\left(h_{n}\right)\right)$ lies in the left kernel of $\hat{\sigma}^{n}(C)$ by Lemma 5.1 (ii).

By Proposition 5.5, $\mathcal{H}_{\infty}$ is trivial iff $C$ has full row rank, which is in agreement with the results in $[23,25]$.

\section{RATIONAL DIFFERENCE EQUATIONS}

The results, obtained in Sections 3, 4 and 5 for the systems described by state equations, that is, by a set of first-order rational difference equations, can be carried over to systems described by input-output equations, that is, by the higher-order rational difference equation.

Let $\mathbf{U}$ be the same as before and $\mathbf{Y}=\left\{y, \sigma(y), \sigma^{2}(y), \ldots\right\}$ be a set of new indeterminates. Extend the automorphism $\sigma: k \rightarrow k$ by mapping $\tilde{u}$ to $\sigma(\tilde{u})$ for all $\tilde{u} \in \mathbf{U}$, and $\tilde{y}$ to $\sigma(\tilde{y})$ for all $\tilde{y} \in \mathbf{Y}$. Then $(k[\mathbf{U}, \mathbf{Y}], \sigma)$ is a ring of difference polynomials. A rational difference equation is of the form

$$
\sigma^{n}(y)=f \quad \text { where } f \in k\left(\mathbf{U}, y, \sigma(y), \ldots, \sigma^{n-1}(y)\right) .
$$

Such an equation is said to be submersive if $\left(\frac{\partial f}{\partial u_{1}}, \ldots, \frac{\partial f}{\partial u_{m}}, \frac{\partial f}{\partial y}\right)$ is nonzero [19]. Write $f$ in (9) as $\frac{d}{c}$, where $c$ and $d$ are coprime in $k\left[\mathbf{U}, y, \sigma(y), \ldots, \sigma^{n-1}(y)\right]$. Set $p=c \sigma^{n}(y)-d$ and $H$ to be the monoid generated by $\sigma^{\ell}(c)$ for all $\ell \in \mathbb{N}$. We call $[p]: H$ the difference ideal associated with (9).
It is well-known that (9) is equivalent to the set of first-order rational difference equations

$$
\left\{\sigma(y)=y_{1}, \ldots, \sigma\left(y_{n-2}\right)=y_{n-1}, \sigma\left(y_{n-1}\right)=\tilde{f}\right\},
$$

where $\tilde{f}$ is obtained by substituting $y_{i}$ for $\sigma^{i}(y)$ in $f$ for $i=1, \ldots$, $n-1$. This equivalence enables us to translate Lemma 3.2, Corollary 3.3 and Proposition 3.4 into the next proposition.

Proposition 6.1 Let I be the difference ideal associated with (9). (i) If I is proper, then $k\left[\mathbf{U}, y, \sigma(y), \ldots, \sigma^{n-1}(y)\right] \cap I=\{0\}$. (ii) $I$ is prime. (iii) I is proper and reflexive iff (9) is submersive. (iv) If (9) is submersive, then $(K, \bar{\sigma})$ is isomorphic to

$$
\left(k\left(\mathbf{U}, y, \sigma(y), \ldots, \sigma^{n-1}(y)\right), \theta\right),
$$

where $K$ is the quotient field of $k[\mathbf{U}, \mathbf{Y}] / I, \bar{\sigma}$ is the map induced by $\sigma, \theta(z)=\sigma(z)$ for $z$ free of $\sigma^{n-1}(y)$, and $\theta\left(\sigma^{n-1}(y)\right)=f$.

An algebraic description of the equivalence between (9) and (10), and a proof of Proposition 6.1 are given in [17].

If (9) is submersive, then we call $(K, \bar{\sigma})$ in Proposition 6.1 the difference field associated with (9). The associated field $K$ of a submersive equation (9) has an inversive closure $(\widehat{K}, \hat{\sigma})$, which is isomorphic to the inversive closure of the field associated with (10).

Next, we translate the results in Section 5 for submersive systems to a submersive equation. This translation leads to a criterion for the accessibility described by input-output equations.

Let (9) be submersive whose associated inversive field is denoted by $(\widehat{K}, \hat{\sigma})$. Let $(\Omega, \mathrm{d})$ be the $\widehat{K}$-linear space of $k$-differentials, which is a module over $S=\widehat{K}[s ; \hat{\sigma}]$. Since $\widehat{K}$ is isomorphic to the inversive field associated with (10), Lemma 5.1 translates into

Lemma 6.2 (i) If $\omega$ is a nonzero element in $\Omega$, then $s \omega \neq 0$.

(ii) In $\Omega,\left\{\mathrm{d} z \mid z \in \mathbf{U} \cup\left\{y, \sigma(y), \ldots, \sigma^{n-1}(y)\right\}\right\}$ is linearly independent over $\widehat{K}$.

From now on, we assume that $m=1$, i.e. $\mathbf{U}=\left\{u, \sigma(u), \sigma^{2}(u), \ldots\right\}$. Differentiating $\hat{\sigma}^{n}(y)=f$ yields

$$
P \mathrm{~d} y=Q \mathrm{~d} u
$$

where $P=s^{n}-\sum_{j=0}^{n-1} \frac{\partial f}{\partial \sigma^{j}(y)} s^{j}$ and $Q=\sum_{i} \frac{\partial f}{\partial \sigma^{i}(u)} s^{i}$. This compact form enables us to determine tor $(\Omega)$ by Ore polynomials.

The next lemma reveals that (12) is the "minimal" linear relation among $\{\mathrm{d} z \mid z \in \mathbf{U} \cup \mathbf{Y}\}$.

Lemma 6.3 Let $P$ and $Q$ be given in (12). If there exist $P^{\prime}$ and $Q^{\prime}$ in $S$ such that $P^{\prime} \mathrm{d} y=Q^{\prime} \mathrm{d} u$ holds in $\Omega$, then $P^{\prime}=D P$ and $Q^{\prime}=D Q$ for some $D$ in $S$.

Proof. The right-hand division yields $P^{\prime}=D P+R$ with $\operatorname{deg} R$ less than $n$. It follows from $P^{\prime} \mathrm{d} y=Q^{\prime} \mathrm{d} u$ and $D P \mathrm{~d} y=D Q \mathrm{~d} u$ (see (12)) that $R \mathrm{~d} y=\left(Q^{\prime}-D Q\right) \mathrm{d} u$. Since $\operatorname{deg} R<n$. Lemma 6.2 (ii) implies that both $R=0$ and $Q^{\prime}=D Q$. Consequently, $P^{\prime}=D P$.

It is important to remark that the greatest common left divisors (abbreviated as: gcld) of $P$ and $Q$ is well-defined and computable by the left-hand Euclidean algorithm, because $\widehat{K}$ is inversive.

Lemma 6.4 Let $A_{1}, A_{2}, B_{1}, B_{2}$ and $L$ be nonzero elements in $S$. If $A_{1}=L B_{1}, A_{2}=L B_{2}$ and $\operatorname{gcld}\left(B_{1}, B_{2}\right)=1$, then $L=\operatorname{gcld}\left(A_{1}, A_{2}\right)$.

Proof. The (extended) left-hand Euclidean algorithm yields $C_{1}$ and $C_{2}$ in $S$ such that $B_{1} C_{1}+B_{2} C_{2}=1$. So $A_{1} C_{1}+A_{2} C_{2}=L$. It follows that $\operatorname{gcld}\left(A_{1}, A_{2}\right)$ is a left-hand divisor of $L$.

The next proposition connects (12) with $\operatorname{tor}(\Omega)$. 
Proposition 6.5 Let $P$ and $Q$ be given in (12) and $G=\operatorname{gcld}(P, Q)$. Put $P=G \tilde{P}, Q=G \tilde{Q}$ and $\tilde{\omega}=\tilde{P} \mathrm{~d} y-\tilde{Q} \mathrm{~d} u$. Then

(i) $\operatorname{tor}(\Omega)=\left\{\omega \in \Omega \mid s^{\ell} \omega \in\right.$ S $\tilde{\omega}$ for some $\left.\ell \in \mathbb{N}\right\}$.

(ii) $\Omega$ is torsion-free iff $G=1$.

Proof. (i) Since (12) implies $G \tilde{\omega}=0, S \tilde{\omega}$ is a finite-dimensional linear subspace over $\widehat{K}$. Consequently, $S \tilde{\omega}$ is a subset of $\operatorname{tor}(\Omega)$, and, hence, $\left\{\omega \mid s^{\ell} \omega \in S \tilde{\omega}\right.$ for some $\left.\ell \in \mathbb{N}\right\}$ is a subset of $\operatorname{tor}(\Omega)$.

Conversely, let $\omega$ be a nonzero torsion element. Putting $y_{0}=y$ and $u_{0}=u$, we may write $\omega$ as $M^{\prime} \mathrm{d} y_{-\ell}-N^{\prime} \mathrm{d} u_{-\ell}$ for some $\ell \in \mathbb{N}$ and $M^{\prime}, N^{\prime} \in S$. By Lemma 6.2, there are nonzero elements $M, N \in S$ such that $s^{\ell} \omega=M \mathrm{~d} y-N \mathrm{~d} u$. It remains to show that $s^{\ell} \omega$ is in $S \tilde{\omega}$. Since $s^{\ell} \omega$ is in $\operatorname{tor}(\Omega), C s^{\ell} \omega=0$ for some $C \in S$ with $C \neq 0$. So $C M \mathrm{~d} y=C N \mathrm{~d} u$. Then $C M=D P$ and $C N=D Q$ for some $D \in S$ by Lemma 6.3, which implies that

$$
C M=D G \tilde{P} \quad \text { and } \quad C N=D G \tilde{Q} .
$$

By Lemma 6.4 and $\operatorname{gcld}(\tilde{P}, \tilde{Q})=1, D G=\operatorname{gcld}(C M, C N)$. Therefore, $D G=C \tilde{C}$ for some $\tilde{C}$ in $S$, which, together with (13), implies that $M=\tilde{C} \tilde{P}$ and $N=\tilde{C} \tilde{Q}$, and so that $s^{\ell} \omega=\tilde{C} \tilde{\omega} \in S \tilde{\omega}$.

(ii) Note that $G=1$ iff $\tilde{\omega}=0$ by (12). The second assertion follows from the first one and Lemma 6.2 (i).

\section{APPLICATIONS}

We apply the results of previous sections to provide an algebraic condition of accessibility for a rational discrete-time nonlinear system, described either by a set of state equations or by a higher-order input-output difference equation. This enables us to implement the accessibility test in computer algebra systems such as Maple or Mathematica. In addition, we relate the algebraic accessibility condition to the reduced form of the transfer function of the system.

In this section, we let $k$ be the field of real numbers, and $\sigma$ the identity map on $k$. We extend $\sigma$ to $k[\mathbf{U}, \mathbf{X}]$ (resp. $k[\mathbf{U}, \mathbf{Y}]$ ) by mapping $z$ to $\sigma(z)$ for all $z \in \mathbf{U} \cup \mathbf{X}$ (resp. $k[\mathbf{U}, \mathbf{Y}]$ ).

\subsection{State-space representations}

We consider the rational control system of the form

$$
\sigma\left(x_{1}\right)=f_{1}, \ldots, \sigma\left(x_{n}\right)=f_{n} \quad \text { and } \quad y=g
$$

where $f_{1}, \ldots, f_{n}, g$ are in $k(\mathbf{u}, \mathbf{x})$. Let $\Sigma$ stand for the rational difference system in (14), and call $y=g$ an output equation. We assume that $\Sigma$ is submersive, and that $\Omega_{\Sigma}, \mathcal{H}_{\infty}$ and the $\mathcal{H}_{i}$ are the same as those in Section 5.

Following [2], we define the relative degree of $\omega \in \Omega_{\Sigma}$ to be

$$
r=\min \left\{\ell \geq 0 \mid s^{\ell} \omega \notin \mathcal{H}_{1}\right\} .
$$

If such an integer does not exist, then set $r=\infty$. An element $\omega \in \Omega_{\Sigma}$ is called an autonomous one-form if its relative degree is $\infty$. Such a one-form and its shifts by the powers of $s$ do not depend on $\mathrm{d}^{\ell}\left(u_{i}\right)$ for all $\ell \in \mathbb{Z}$ and $i \in\{1, \ldots, m\}$.

Clearly, every element of $\mathcal{H}_{\infty}$ is autonomous. On the other hand, $\omega, s \omega, s^{2} \omega \ldots$ are all in $\mathcal{H}_{1}$ if $\omega$ is autonomous. Hence, $\omega$ belongs to $\mathcal{H}_{\infty}$ by Proposition 5.4. Accordingly, $\mathcal{H}_{\infty}$ is precisely the set of autonomous elements.

We say that (14) is accessible if $\Omega_{\Sigma}$ does not contain any nontrivial autonomous one-form, that is, $\mathcal{H}_{\infty}$ is trivial (see [2, Theorem 4.5]). By Proposition 5.3, (14) is accessible iff $\Omega_{\Sigma}$ is torsion-free. The latter condition can be verified by computing the rank of the matrix $C$ defined in Proposition 5.5.
Example 7.1 Consider the rational control system of the form

$$
\sigma\left(x_{1}\right)=x_{2} u, \sigma\left(x_{2}\right)=x_{1} u \text { and } y=x_{1}^{2}+x_{1} .
$$

The difference field associated with (15) is isomorphic to

$$
K=\left(k\left(x_{1}, x_{2}, u, \sigma(u), \ldots\right), \theta\right),
$$

where $\theta\left(\sigma^{i}(u)\right)=\sigma^{i+1}(u), \theta\left(x_{1}\right)=x_{2} u$, and $\theta\left(x_{2}\right)=x_{1} u$. The inversive closure of $K$ is $\widehat{K}=\left(k\left(x_{1}, x_{2}, \ldots, \sigma^{-1}(u), u, \sigma(u), \ldots\right), \hat{\theta}\right)$, where $\hat{\theta}^{-1}\left(x_{1}\right)=\frac{x_{2}}{\sigma^{-1}(u)}, \hat{\theta}^{-1}\left(x_{2}\right)=\frac{x_{1}}{\sigma^{-1}(u)}$, and $\hat{\theta}^{-1}\left(\sigma^{i}(u)\right)=\sigma^{i-1}(u)$ for all $i \in \mathbb{Z}$. Then (7) becomes

$$
s\left(\begin{array}{l}
\mathrm{d} x_{1} \\
\mathrm{~d} x_{2}
\end{array}\right)=\left(\begin{array}{ll}
0 & u \\
u & 0
\end{array}\right)\left(\begin{array}{l}
\mathrm{d} x_{1} \\
\mathrm{~d} x_{2}
\end{array}\right)+\left(\begin{array}{l}
x_{2} \\
x_{1}
\end{array}\right) \mathrm{d} u .
$$

The matrix $C$ given in Proposition 5.5 is

$$
\left(\begin{array}{cc}
x_{1} / \sigma^{-1}(u) & x_{1} / \sigma^{-2}(u) \\
x_{2} / \sigma^{-1}(u) & x_{2} / \sigma^{-2}(u)
\end{array}\right) .
$$

The left kernel of $C$ is spanned by $\left(x_{2},-x_{1}\right)$. Hence, $\mathcal{H}_{\infty}$ is spanned by $x_{2} \mathrm{~d} x_{1}-x_{1} \mathrm{~d} x_{2}$ over $\widehat{K}$. So (15) is not accessible.

Differentiating the equation $y=x_{1}^{2}+x_{1}$ yields $\mathrm{d} y=\left(2 x_{1}+1\right) \mathrm{d} x_{1}$, which, together with (16), enables us to express sdy and $s^{2} \mathrm{~d} y$ as a linear combination of $\mathrm{d} u, \mathrm{~d} x_{1}$ and $\mathrm{d} x_{2}$. Using the three linear relations to eliminate $\mathrm{d} x_{1}$ and $\mathrm{d} x_{2}$, we find that $P \mathrm{~d} y=Q \mathrm{~d} u$ holds in $\Omega_{\Sigma}$, where $P=s^{2}-\frac{2 x_{1} u^{2} \sigma(u)^{2}+u \sigma(u)}{2 x_{1}+1}$ and

$$
Q=\left(2 x_{1} u \sigma(u)+1\right) x_{1} u s+\left(2 x_{1} u \sigma(u)+1\right) x_{1} \sigma(u) .
$$

The skew-fraction $P^{-1} Q$ is called the transfer function of $\Sigma([15])$. We find that $\operatorname{gcld}(P, Q)$ has degree one by left-hand Euclidean algorithm in $S_{\Sigma}$. Hence, the reduced transfer function equals $\tilde{P}^{-1} \tilde{Q}$, where $\tilde{P}=s-\frac{x_{2} u\left(2 x_{2} u+1\right)}{x_{1}\left(2 x_{1}+1\right)}$ and $\tilde{Q}=x_{2}+2 u x_{2}^{2}$.

For this example, one can use algebraic Gröbner basis computation to get $x_{1}=\frac{y u^{2} \sigma(u)^{2}-\sigma^{2}(y)}{u \sigma(u)(u \sigma(u)-1)}$ and $x_{2}=\frac{\sigma(y)(\sigma(u))^{2}\left(\sigma^{2}(u)\right)^{2}-\sigma^{3}(y)}{u \sigma(u) \sigma^{2}(u)\left(\sigma(u) \sigma^{2}(u)-1\right)}$. Therefore, the reduced transfer function can be expressed as a lefthand skew-fraction whose coefficients are rational functions in the input and output variables.

\subsection{Input-output difference equations}

Let $\mathbf{U}=\{u, \sigma(u), \ldots\}$ and $\mathbf{Y}=\{y, \sigma(y), \ldots\}$. For brevity, we put $\sigma^{i}(y)=y^{[i]}, \sigma^{j}(u)=u^{[j]}$ for $i, j \in \mathbb{Z}$. Consider a single-input single-output equation of the form

$$
y^{[n]}=f\left(u^{[0]}, u^{[1]}, \ldots, u^{[n-1]}, y^{[0]}, y^{[1]}, \ldots, y^{[n-1]}\right) .
$$

We assume that (17) is submersive, and that $(\widehat{K}, \hat{\sigma})$ and $(\Omega, \mathrm{d})$ are the same as those in Section 6.

Following [19], we define the relative degree of $\omega \in \Omega$ to be

$$
r=\min \left\{\ell \geq 0 \mid s^{\ell} \omega \notin \mathcal{G}_{1}\right\},
$$

where $\mathcal{G}_{1}=\operatorname{span}_{\widehat{K}}\left\{\mathrm{~d} y^{[0]}, \ldots, \mathrm{d} y^{[n-1]}, \mathrm{d} u^{[0]}, \ldots, \mathrm{d} u^{[n-1]}\right\}$. If such an integer does not exist, then set $r=\infty$. An element $\omega \in \Omega$ is called an autonomous one-form if its relative degree is $\infty$. Such a one-form and its shifts by the powers of $s$ do not depend on $\mathrm{d} u^{[i]}$ for all $i \geq n$. An autonomous one-form $\omega$ is a torsion element, since the submodule $S \omega \subset G_{1}$ is a finite-dimensional linear space over $\widehat{K}$.

We say that (17) is accessible if $\Omega$ does not contain any nontrivial autonomous one-form, that is, $\Omega$ is torsion-free. The latter condition can be verified by determining whether $P$ and $Q$ given by (12) have a trivial gcld by Proposition 6.5. 
The left-hand skew-fraction $P^{-1} Q$ is called the transfer function of (17) in [16]. To find its reduced form, one has to compute the gcld of $P$ and $Q$. A Maple package is described in [26] for computing transfer functions. An idea for modular gcld-calculation in $\widehat{K}[s ; \hat{\sigma}]$ is outlined in [22].

Example 7.2 Consider a submersive equation

$$
y^{[4]}=y^{[0]} y^{[1]} u^{[2]}+u^{[0]} u^{[1]} y^{[3]}+u^{[0]} y^{[2]} u^{[3]}
$$

whose associated inversive field is denoted by $\widehat{K}$. Using (12), we find that $P \mathrm{~d} y=Q \mathrm{~d} u$ holds in the module $\Omega$ of Kähler differentials corresponding to the equation, where

$$
\begin{aligned}
& P=s^{4}-u^{[0]} u^{[1]} s^{3}-u^{[0]} u^{[3]} s^{2}-y^{[0]} u^{[2]} s-y^{[1]} u^{[2]} \\
& Q=u^{[0]} y^{[2]} s^{3}+y^{[0]} y^{[1]} s^{2}+u^{[0]} y^{[3]} s+u^{[1]} y^{[3]}+y^{[2]} u^{[3]}
\end{aligned}
$$

To avoid using the left Euclidean algorithm to compute $\operatorname{gcld}(P, Q)$, which may cause intermediate expressions to swell, we use the notion of (right-hand side) Sylvester resultants. Let $P_{a}$ and $Q_{a}$ be the adjoint operators of $P$ and $Q$, respectively (see, e.g. [1]). Note that $P_{a}$ and $Q_{a}$ are in $\widehat{K}\left[s ; \hat{\sigma}^{-1}\right]$. Then $\operatorname{gcld}(P, Q)=1$ iff the Sylvester resultant of $P_{a}$ and $Q_{a}$ is nonzero [21].

Form the Sylvester resultant $D$ of the respective numerators of $P_{a}$ and $Q_{a}$ to get a $7 \times 7$ determinant whose entries are polynomials in $y^{[-6]}, y^{[-5]}, y^{[-4]}, y^{[-3]}, u^{[-6]}, u^{[-5]}, \ldots$ over $k$. Computing the images of the entries of $D$ modulo 2 , and setting $u^{[i]}=1$ and $y^{[j]}=1$ for $i=-6,-5, \ldots$, and $j=-6, \ldots,-3$, we get a $7 \times 7$ nonzero determinant over $\mathbb{Z}_{2}$. So $\operatorname{gcld}(P, Q)=1$, and (18) is accessible.

We cannot assert that $D$ is zero when a homomorphic image of $D$ vanishes. However, we may estimate the degree and coefficient bounds of $D$, and decide if it is zero using sufficiently many modular and evaluation homomorphisms.

Acknowledgment: We thank Martin Ondera for initiating our collaboration and for his helpful comments.

\section{REFERENCES}

[1] S.A. Abramov, H.Q. Le and Z. Li. Univariate Ore polynomial rings in computer algebra. Journal of Mathematical Sciences, 131(5), 5885-5903, 2005.

[2] E. Aranda-Bricaire, Ü. Kotta and C.H. Moog. Linearization of discrete-time systems. SIAM J. Control and Optimization, 34(6), 1999-2023, 1996.

[3] M. Bronstein. Symbolic Integration I: Transcedental Functions, second edition, Springer-Verlag, 2005.

[4] M. Bronstein and M. Petkovšek. An introduction to pseudo-linear algebra. Theoretical Computer Science, 157(1), 3-33, 1996.

[5] H. Cheng and G. Labahn. Output-sensitive modular algorithms for polynomial matrix normal forms. Journal of Symbolic Computation, 42(7), 733-750, 2007.

[6] F. Chyzak, A. Quadrat and D. Robertz. Effective algorithms for parametrizing linear control systems over Ore algebras. Applicable Algebra in Engineering, Communications and Computing, 16(5), 319-376, 2005.

[7] P.M. Cohn. Free Rings and Their Relations. Academic Press, London, 1985.

[8] R.M. Cohn. Difference Algebra. Interscience Publishers, John Wiley, New York, 1965.

[9] G. Conte, A.M. Perdon, C.H. Moog. The differential field associated to a general analytic nonlinear dynamic system. IEEE Trans on Automatic Control, 38(7), 1120-1124, 1993.
[10] M. Fliess, C. Join and H. Sira-Ramírez. Non-linear estimation is easy. International Journal of Modelling, Identification and Control, 4(1), 12-27, 2008.

[11] M. Fliess and H. Mounier. Controllability and obesrvability of linear delay systems: an algebraic approach. ESIAM: Control, Optimisation and Calculus of Variations, $\mathbf{3}$, 301-314, 1998.

[12] X.S. Gao, J. van der Hoeven, C.M. Yuan, and G. Zhang. Characteristic set method for differential-difference polynomial systems. Journal of Symbolic Computation, doi:10.1016/j.jsc.2008.02.010.

[13] J.W. Grizzle. A linear algebraic framework for the analysis of discrete-time nonlinear systems. SIAM J. Control and Optimazation, 31(4), 1026-1044, 1993.

[14] M. Halás. An algebraic framework generalizing the concept of transfer functions to nonlinear systems. Automatica, 44(5), 1181-1190, 2008.

[15] M. Halás and Ü. Kotta. Pseudo-linear algebra: a powerful tool in unification of the study of nonlinear control systems. In Proc. of 7th IFAC Symposium NOLCOS, 2007.

[16] M. Halás and Ü. Kotta. Transfer functions of discrete-time nonlinear control systems. Proc. of Estonian Academy of Science. Physics. Mathematics, 56(4), 322-335, 2007.

[17] M. Halás, Ü. Kotta, Z. Li, H. Wang and C. Yuan. Submersive rational difference system and formal accessibility. Math. Mech. Research Preprints, 28, 40-57, 2009. (www.mmrc.iss.ac.cn/mmpreprints).

[18] J. Johnson. Kähler differentials and differential algebra. Annals of Mathematics, 89(1), 92-98, 1969.

[19] Ü. Kotta. Irreducibility conditions for nonlinear input-output difference equations. In Proc. of the 39th IEEE Conf. on Decision and Control, 4, 3404-3408, 2000.

[20] Ü. Kotta, A. Zinober and P. Liu. Transfer equivalence and realization of nonlinear higher order input-output difference equations. Automatica, 37(11), 1771-1778, 2001.

[21] Z. Li and I. Nemes. A modular algorithm for computing greatest common right divisors of Ore polynomials. In Proc. of 1997 International Symposium on Symbolic and Algebraic Computation, 282-289, ACM Press, 1997.

[22] Z. Li, M. Ondera and H. Wang. Simplifying skew fractions modulo differential and difference relations. A poster presented at the 2008 International Symposium on Symbolic and Algebraic Computation, RISC-Linz, Austria, 2008.

[23] L.A. Márquez-Martínez. Note sur l'accessibilité des systèmes non linéaires à retards. Comptes Rendus de l'Academie des Sciences Paris, 329, 545-550, 1999.

[24] S. Morrison. The differential ideal $P: M^{\infty}$. Journal of Symbolic Computation, 28(4-5), 631-656, 1999.

[25] T. Mullari, Ü. Kotta and M. Tõnso. The connection between different static state feedback linearizability conditions of discrete time nonlinear control systems. In Proc. of European Control Conference, 4268-4275, 2007.

[26] M. Ondera. GTF_Tools: A computer algebra package for generalized transfer functions. In Proc. of the 15th Mediterranean Conference on Control and Automation, Athens, Greece, 2007.

[27] O. Ore. Theory of non-commutative polynomials. Annals of Mathematics, 34(22), 480-508, 1933.

[28] M. Rosenlicht. On Liouville's theory of elementary functions. Pacific J. Math., 65(2), 485-492, 1976. 